\title{
Rapid Analysis \& Design Methodologies of High- Frequency LCLC Resonant Inverter as Electrodeless Fluorescent Lamp Ballast
}

\author{
Yong-Ann Ang, David Stone, Chris Bingham, Martin Foster \\ Dept of Electronic Engineering, University of Sheffield, Mappin Street, Sheffield, S1 3JD. UK. (0)114 2225046. \\ d.a.stone@sheffield.ac.uk
}

\begin{abstract}
The papers presents methodologies for the analysis of $4^{\text {th }}$-order LCLC resonant power converters operating at $2.63 \mathrm{MHz}$ as fluorescent lamp ballasts, where high frequency operation facilitates capacitive discharge into the tube, with near resonance operation at high load quality factor enabling high efficiency. State-variable dynamic descriptions of the converter are employed to rapidly determine the steady-state cyclic behaviour of the ballast during nominal operation. Simulation and experimental measurements from a prototype ballast circuit driving a $60 \mathrm{~cm}$, 8W T5 fluorescent lamp are also included.
\end{abstract}

\section{INTRODUCTION}

Fluorescent lighting takes a major role in today's lighting requirements (with around 1.2 billion units being produced per year) due to benefits afforded by crisp white light output compared to traditional incandescent and high intensity discharge lamps. Fluorescent lamps also provide a higher Lumens/Watt output, and higher efficiency, particularly when excited at high frequencies, typically $30-$ $50 \mathrm{kHz}$, by virtue of there being insufficient time between each half cycle of the supply for a significant number of mercury ions in the discharge to re-combine (and thereby necessitating a re-strike), as occurs with standard mains frequency excitation, for instance.

The affect of this non-linear frequency dependence of lamp voltage and current can be clearly seen from a comparison of Figs. 1(a) \& 1(b), which show the voltage vs. current relationship for an $8 \mathrm{~W}, 60 \mathrm{~cm}$, T5 fluorescent tube excited by $50 \mathrm{~Hz}$ and $50 \mathrm{kHz}$ input voltage, respectively.

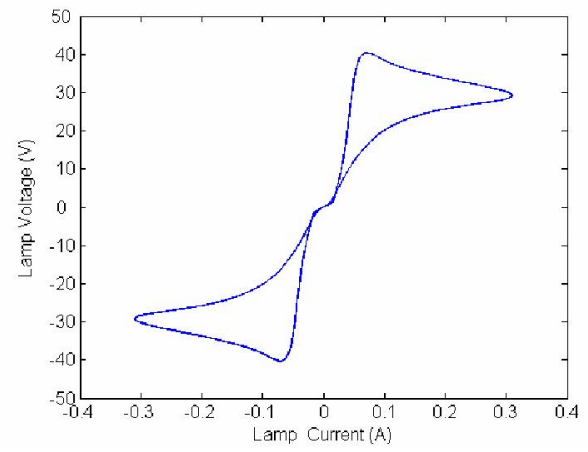

(a)

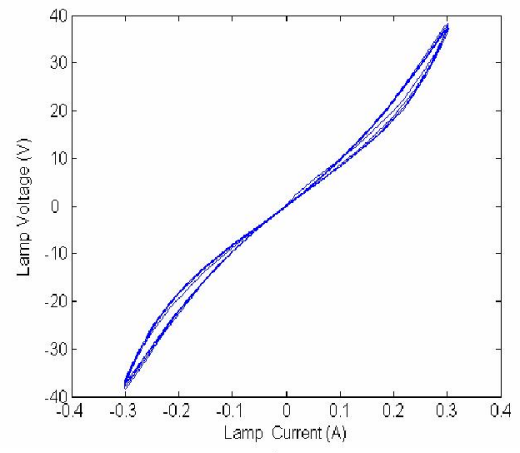

(b)

Figure 1 Lamp voltage vs. current (a) $50 \mathrm{~Hz}$ excitation (b) $50 \mathrm{kHz}$ excitation

The relative 'loop area' shows that less re-combination occurs within the lamp between each half cycle of the input voltage when high frequency excitation is employed. Typically, fluorescent lamps are constructed with an oxide coated, tungsten filament electrode. Passing current through the electrode prior to striking to heat it (termed preheating) lowers the electrode work function, thereby allowing electrons to be emitted more readily. This consequently allows the lamp to strike at lower voltage than would normally be required, which in-turn reduces damage to the electrode from ion bombardment during the ignition event. Despite electrode pre-heating, however, the most common lamp failure mechanism is due to breakdown of the coating on the electrodes, giving rise to a blackening at one end of the tube and the lamp subsequently acting with similar characteristics to that of a gas diode. To circumvent this problem, and ultimately increase lamp lifetime, electrode-less lamps have been developed with various techniques being employed to sustain the arc viz. RF induction and capacitive discharge [1], and usually require excitation frequencies in the $\mathrm{MHz}$ range. Along with increasing lifetime, electrode-less excitation also removes the loss associated with the electrode heating, and therefore encourages higher operating efficiency. Such lamps (for example the GE Genura $23 \mathrm{~W}$ commercially available induction coupled lamp) are increasingly becoming a preferred candidate for inaccessible environments viz: high ceiling sports halls requiring low maintenance etc. A demonstrator capacitively coupled lamp, $8 \mathrm{~W}, 60 \mathrm{~cm}, \mathrm{~T} 5$ fluorescent tube coupled to a ballast via copper tape applied to the outside of the tube, is shown in Fig. 2(a). To provide 
ignition and sustained light output from the lamp, an electronic ballast must develop sufficiently high voltage, typically between $400 \mathrm{~V}$ and $1000 \mathrm{~V}$ for striking, and subsequently provide current limiting to promote stable operation. The latter issue is a key motivator for adopting resonant converters for fluorescent lamp ballasts, since, after striking, the lamp exhibits a negative incremental impedance characteristic, as shown in Fig. 2(b) \& (c) for an $8 \mathrm{~W}$ lamp with an ignition voltage of $270 \mathrm{~V}$. The series impedance of resonant converters naturally acts to counter this destabilizing characteristic and encourage steady continuous operation. When operating without electrodes, the requirement for high frequency excitation also has the additional advantage of reducing the volume envelope requirements of the reactive components of the ballast, although this is at the expense of significantly complicating the design of the ballast since circuit behaviour can become dominated by parasitic elements.

Electronic ballasts with half-bridge series resonant inverters are relatively straightforward to design, and have been widely reported e.g $[2,3,4]$, along with the more complicated $3^{\text {rd }}$-order LCC inverter variants [5]. More generically, however, for applications that are battery powered or require battery backup facilities, for instance, a low DC input voltage must be 'boosted' for lamp ignition through the incorporation of a step-up transformer [6].

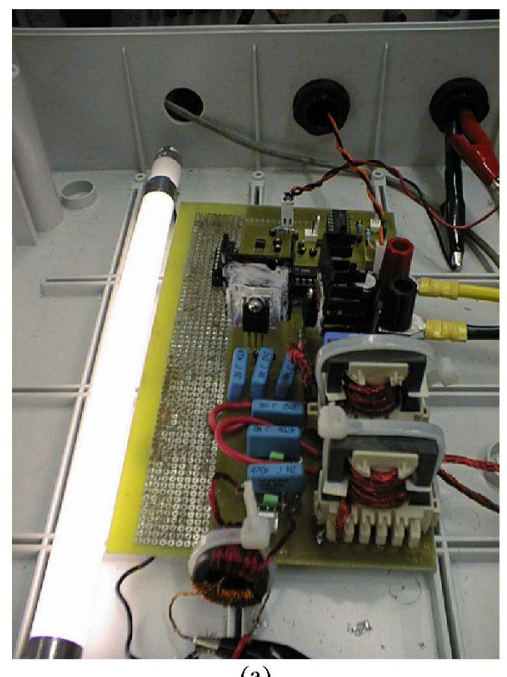

(a)

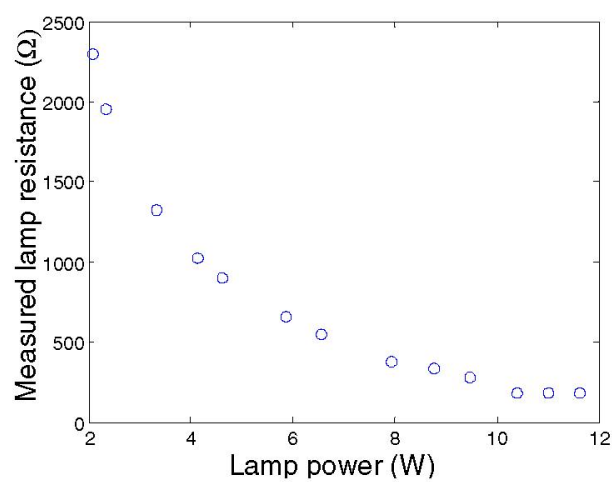

(b)

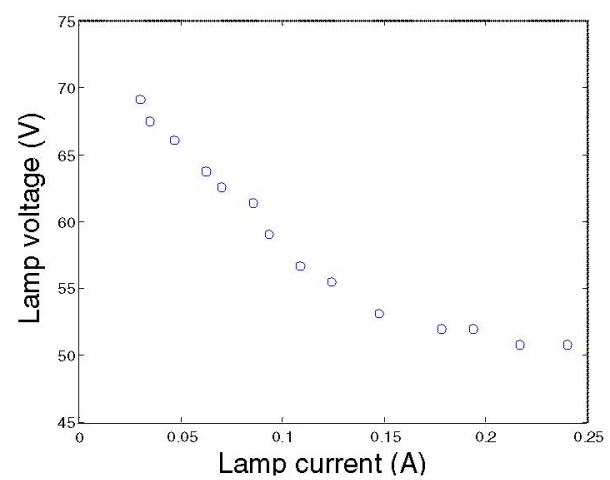

(c)

Figure 2 Capacitively coupled fluorescent lamp (a) Lamp and inverter (b) Measured lamp resistance against power (c) Measured voltage and current characteristics

The subsequent effects of the high-frequency transformer's magnetising inductance and inter-winding capacitance, are then best represented by a model of a $4^{\text {th }}$-order LCLC resonant inverter. The widespread adoption of such highorder resonant inverters, however, has been impeded by the higher peak electrical stresses to which individual electronic components are exposed (compared to hardswitched inverter counterparts), and the lack of suitable design methodologies that can provide an accurate and rapid analysis of the circuit at the design stage; particularly those that consider the significant effects that parasitic resistances, capacitances and inductances have on the resonant tank behaviour.

These difficulties have prompted researchers to investigate techniques for the reliable analysis and design of resonant inverters. Time domain mathematical models used to describe compact fluorescent ballasts have previously been reported in [7, 8], where theoretical results of seriesresonant and series-parallel LCC electronic ballasts are shown to provide good agreement with measured data, at the expense of requiring significant computation overhead. Moreoften therefore, designers return to Fundamental Mode Analysis (FMA) [9, 10] for simplifying and speeding-up the design and analysis process, at the expense of neglecting the important harmonic and sub-harmonic content of the circuit voltages and currents.

Here then, the paper considers cyclic analysis $[11,12,13$, 14] as a candidate technique for the rapid analysis and design of $4^{\text {th }}$-order LCLC resonant inverters, suitable for use as electronic ballasts for example, including a treatment of parasitic effects. The main aim of the full paper is therefore to address the lack of suitable design methodologies for high order, high frequency resonant converters, the lamp providing a suitable candidate application for exploitation and verification of the work. By suitable manipulation, the resulting models are then employed to analytically predict the voltage and current stresses on the resonant components, with measurements from a prototype $8 \mathrm{~W}, 4^{\text {th }}$-order, capacitively coupled ballast used to demonstrate the accuracy of the model predictions. 


\section{ANALYSIS OF ELECTRODE-LESS FLOURESCENT- LAMP BALLAST}

As previously discussed, suitable $4^{\text {th }}$-order electronic ballasts must provide sufficient voltage to promote ignition, and a current limiting capability thereafter. Before the lamp is ignited, it can be assumed that the resistance presented to the output of the ballast is infinite i.e. the output stage is open circuit. Consequently, the resonant inverter behaves as a tank circuit with a high effective $Q$ to facilitate ionization of the gas within the tube. Of note, is that the minimum capacitive discharge voltage decreases with increasing frequency, when the capacitive coupling reactance becomes small. Once gaseous breakdown has occurred, the resistance decreases as the lamp conducts current. From Fig.2(b), the nominal resistance of the lamp during normal $(8 \mathrm{~W})$ operation can, for this case, be estimated to be $\cong 470 \Omega$. An equivalent circuit of the system therefore consists of a $4^{\text {th }}$-order LCLC resonant inverter loaded by the lamp resistance, $R_{\text {lamp }}$, see Fig.3.

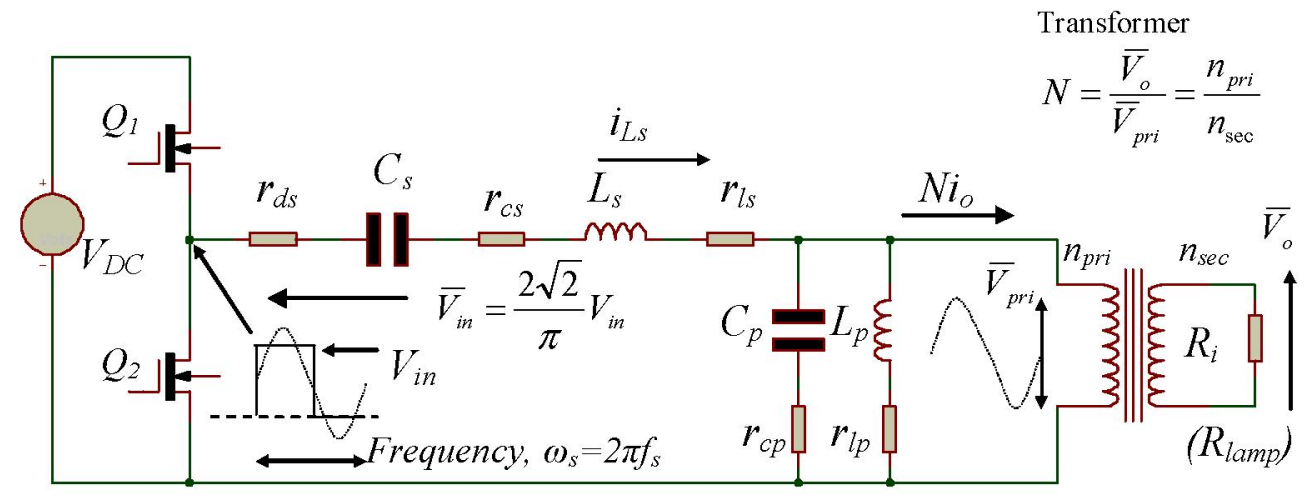

Figure 3 LCLC resonant inverter for fluorescent lamp ballast $\left(R_{\text {laupp }}\right)$

State Variable Modelling

Figure 3 depicts the structure of a $4^{\text {th }}$-order LCLC resonant power inverter. In high frequency inverters, such as those for fluorescent lamp ballast applications, the parallel resonant components, $L_{p}$ and $C_{p}$, are designed to be the magnetising inductance and parasitic capacitance of a stepup transformer, whilst the series resonant component, $L_{s}$, takes advantage of the transformer leakage inductance. In this way, the high order circuit is achieved with few additional passive components. Parasitic circuit elements have also been included in Fig. 3 for completeness. A statevariable dynamic model of the circuit can be derived by considering the resonant tank components and power switches:

$$
\begin{aligned}
& \frac{d V_{C p}}{d t}=\frac{i_{L S}-i_{L p}-N \cdot i_{O}}{C_{p}} \\
& \frac{d V_{C s}}{d t}=\frac{i_{L S}}{C_{S}} \\
& \frac{d i_{L p}}{d t}=\frac{V_{C p}-i_{L p} \cdot\left(r_{C p}+\eta_{p}\right)+r_{C p} \cdot i_{L S}-N \cdot r_{C p} \cdot i_{O}}{L_{p}} \\
& \frac{d i_{L S}}{d t}=\frac{V_{i n}-V_{C S}-V_{C p}-i_{L S} \cdot\left(r_{d s}+r_{C p}+r_{C S}+r_{s}\right)+r_{C p} \cdot i_{L p}+N \cdot r_{C p} \cdot i_{O}}{L_{S}}
\end{aligned}
$$

with the output voltage $V_{o}$ and load current $i_{o}$ given by:

$$
V_{o}=N \cdot\left[V_{C p}+r_{c p} \cdot\left(i_{L s}-i_{L p}-N \cdot i_{o}\right)\right], \quad i_{o}=N \cdot\left[\frac{V_{C p}}{\left(R_{i}+N \cdot r_{c p}\right)}+\frac{r_{c p} \cdot\left(i_{L s}-i_{L p}\right)}{\left(R_{i}+N \cdot r_{c p}\right)}\right]
$$

The proposed state-variable model is therefore given by:

$$
\left[\begin{array}{c}
\dot{V_{C p}} \\
\dot{V}_{C s} \\
\dot{i}_{L p} \\
i_{L s}
\end{array}\right]=\left[\begin{array}{cccc}
0 & 0 & -\frac{1}{C_{p}} & \frac{1}{C_{p}} \\
0 & 0 & 0 & \frac{1}{C_{s}} \\
\frac{1}{L_{p}} & 0 & -\left(\frac{r_{c p}+r_{p p}}{L_{p}}\right) & \frac{r_{c p}}{L_{p}} \\
-\frac{1}{L_{s}} & -\frac{1}{L_{s}} & \frac{r_{c p}}{L_{s}} & -\left(\frac{r_{d s}+r_{c s}+r_{c p}+r_{l s}}{L_{s}}\right)
\end{array}\right] \cdot\left[\begin{array}{c}
V_{C p} \\
V_{C s} \\
i_{L p} \\
i_{L s}
\end{array}\right]+\left[\begin{array}{c}
-N\left(\frac{i_{o}}{C_{p}}\right) \\
0 \\
-N\left(\frac{r_{c p} \cdot i_{o}}{L_{p}}\right) \\
\frac{V_{i n}}{L_{s}}+N\left(\frac{r_{c p} \cdot i_{o}}{L_{s}}\right)
\end{array}\right]
$$

To demonstrate the accuracy of the model, an experimental $4^{\text {th }}$-order LCLC inverter $\left(V_{D C}=12 \mathrm{~V}\right)$ is considered, as required for example for battery backup lighting applications. A step-up toroidal transformer with primary to secondary turn ratio $n=0.5$ is incorporated to demonstrated the ability of the higher order resonant circuit to take advantage of the transformer magnetising inductance. The resonant frequency of the tank circuit is highly sensitive to variations in component values, and whilst $C_{s}$ and $C_{p}$ can normally be assumed to be within standard component tolerances, the inter-turn capacitance $\left(C_{T}\right)$ of $L_{s}$ and $L_{p}$ must be accommodated. For example, the inter-turn capacitance of $L_{p}$ is measured as $C_{T} \approx 30 p F$ at self-resonance. The resonant inductor and the inter-turn capacitance constitute a parallel resonant circuit, which resembles an equivalent inductance when excited below the combined resonant frequency. A method of accommodating these effects is to define $L_{s}$ and $L_{p}$ as frequency-dependent equivalent inductances, see Fig. 4.

The frequency-dependent equivalent inductance, $L_{p e q}$, is then obtained from the parallel combination of $L_{p} \& C_{T}$ :

$$
Z_{e q}=\frac{j \omega_{s} L_{p}}{1-\omega_{s}^{2} L_{p} C_{T}}=j \omega_{s} L_{p e q}, \quad \text { where } L_{p e q}=\frac{L_{p}}{1-\omega_{s}{ }^{2} L_{p} C_{T}}
$$




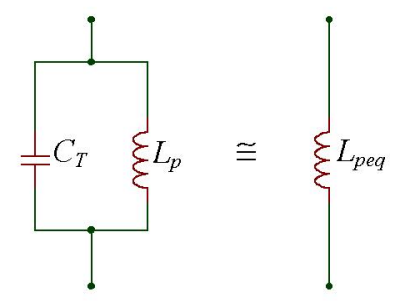

Figure 4 Equivalent circuit of resonant inductor and stray capacitance

After inclusion of the equivalent inductance (4) in the dynamic model, the measured output voltage of the inverter is compared with that predicted from the proposed statevariable model, and Spice simulations, over a range of operating frequencies and output loads, including the opencircuit condition are shown in Fig. 5. It can be seen that the proposed model provides commensurate accuracy with results obtained from Spice.

\section{PRINCIPLES OF CYCLIC ANALYSIS}

Although the presented state-variable description has been shown to accurately model the behaviour of the $4^{\text {th }}$ order inverter, the computation time remains prohibitive due to the requirement for integration, and impedes the use of such models as an interactive design tool. This drawback can be abated to some degree however by only considering the steady-state behaviour of the circuit, thereby allowing analytical solutions from the state equations to be obtained for investigative and design validation purposes.

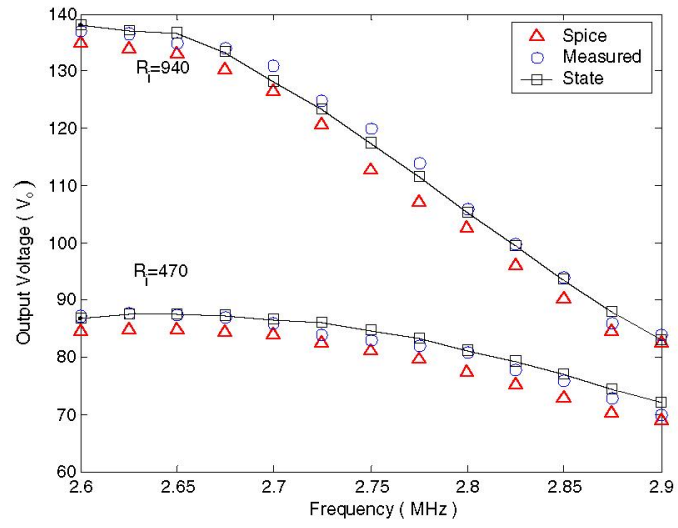

Figure 5 Output voltage of the $4^{\text {th }}$-order resonant inverter at $R_{i}=470 \Omega$ and, $R_{i}=940 \Omega$

In particular, it is now shown that cyclic-modelling [11, 13, 14] provides a convenient methodology to facilitate the rapid solution of the steady state voltages and currents for the $4^{\text {th }}$-order ballast.

\section{Cyclic Modes}

An electronic circuit is said to operate in a cyclic-mode when the state vector $x(t)$ at any time $t$ is equal to $x(t+n T)$, where $T$ is the switching period of the converter and $n$ is an integer, i.e. $x(t+n T)=x(t)$. For a resonant inverter, each cycle is comprised of multiple operating modes, $m_{i}$, each dependent on the state of the input voltage. When considering operation in a cyclic-mode, a system of piecewise linear (state-space) equations that describe the inverter in each operational mode during a cycle can be derived, viz.:

$$
\dot{x}_{i}=\mathbf{A}_{i} x_{i}+\mathbf{B}_{i}
$$

where $x_{i}$ is the state vector, $\mathrm{A}_{i}$ represents the dynamics and $\mathrm{B}_{i}$ is the excitation matrix during the $i^{\text {th }}$-operating mode. For the $i^{\text {th }}$ mode, (5) can be solved analytically to give:

$$
x_{i}(t)=e^{\mathbf{A}_{i} t} x_{i}\left(t_{0}\right)+\int_{0}^{t} e^{\mathbf{A}_{i}(t-\tau)} \mathbf{B}_{i} d \tau=\Phi_{i} x_{i}\left(t_{0}\right)+\Gamma_{i}
$$

where $\Phi_{i}=\Phi\left(t, t_{0}\right)=e^{\mathbf{A}_{i} t}, \Gamma_{i}=\int_{0}^{t} e^{\mathbf{A}_{i}(t-\tau)} \mathbf{B}_{i} d \tau$, and $x_{i}\left(t_{0}\right)$ are the initial conditions for the $i^{\text {th }}$ mode. By noting that the time during which the circuit operates in the $i^{\text {th }}$ mode is $d_{i} T$, where $d_{i}$ is the duty, the complete solution for the system can be obtained by employing the state vector at time $d_{i} T$ as the initial condition for the subsequent dynamics of the $(i+1)^{\text {th }}$ mode. However, the need to evaluate the integral in (6) is a key cause of the computational overhead when analysing the system in this manner. By combining $\mathrm{A}_{i}$ and $\mathrm{B}_{i}$ to form an augmented dynamics matrix, (7), the integration overhead can be eliminated at the expense of obtaining only the 'cyclic' steady-state description:

$$
\frac{d}{d t}\left(\frac{x_{i}(t)}{1}\right)=\left(\begin{array}{c|c}
\mathbf{A}_{i} & \mathbf{B}_{i} \\
\hline 0 & 0
\end{array}\right)\left(\begin{array}{c}
x_{i}(t) \\
\mathbf{1}
\end{array}\right), \quad \text { or } \quad \frac{d}{d t} \hat{x}_{i}(t)=\hat{\mathbf{A}}_{i} \hat{x}_{i}(t)
$$

Now, if mode 1 corresponds to the time period between $t_{0}$ and $t_{i}$, and mode 2 corresponds to the time period between $t_{1}$ and $t_{2}$ the solution for the state vector at the transition time between modes $1 \& 2, t_{1}$, is given by $\hat{x}_{1}\left(t_{1}\right)=e^{\hat{\mathbf{A}}_{1} d_{1} T} \hat{x}_{1}\left(t_{0}\right)=\hat{\Phi}_{1} \hat{x}_{1}\left(t_{0}\right)$. Similarly, the state vector at the transition time between modes 2 and $3, t_{2}$, is $\hat{x}_{2}\left(t_{2}\right)=e^{\hat{\mathbf{A}}_{2} d_{2} T} \hat{x}_{2}\left(t_{0}\right)=\hat{\boldsymbol{\Phi}}_{2} \hat{\boldsymbol{\Phi}}_{1} \hat{x}_{1}\left(t_{0}\right)$. In general, for the $m^{\text {th }}$ mode:

$$
\hat{x}\left(t_{m}\right)=\hat{\Phi}_{m} \hat{\Phi}_{m-1} \cdots \hat{\Phi}_{1} \hat{x}\left(t_{0}\right)=\hat{\Phi}_{t o t} \hat{x}\left(t_{0}\right)
$$

where $\hat{\Phi}_{i}=\left(\begin{array}{c|c}\Phi_{i} & \Gamma_{i} \\ \hline 0 & 1\end{array}\right)$, and $\hat{x}\left(t_{m}\right)$ is the state-vector at time $t_{m}$ for an initial condition $\hat{x}\left(t_{0}\right)$, and, therefore, by definition of the cyclic mode, is equivalent to the initial condition for the cyclic solution. Since behaviour in the cyclic mode necessarily presumes periodic steady-state operation, the initial condition for operation in a cyclic mode is given by,

$$
x_{\text {per }}\left(t_{0}\right)=\left(I^{n}-\Phi_{\text {tot }}\right)^{-1} \Gamma_{\text {tot }}=\hat{x}\left(t_{0}\right)
$$

from which the initial condition for the voltage and current of the inverter and, together with (8) can be used to determine the voltage and current state at subsequent times.

\section{OPERATION OF LCLC INVERTER IN A CYCLIC MODE}

For operation above resonance, which is the norm, analysis of the behaviour of the $4^{\text {th }}$-order LCLC inverter identifies 6 modes of operation within each periodic cycle. 


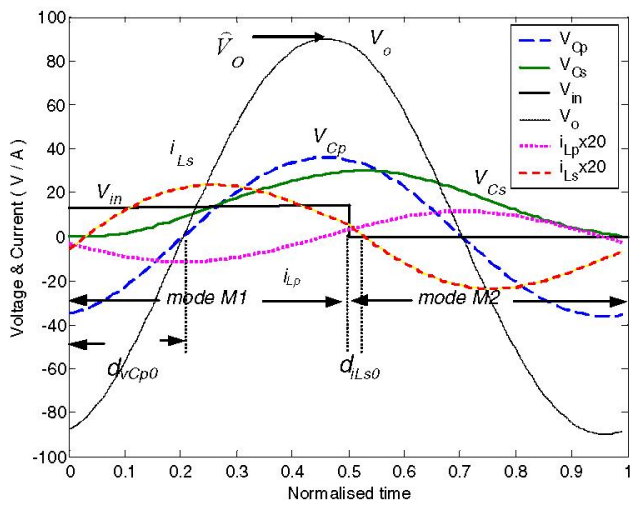

Figure 6 Voltage and current waveforms and dominant operating modes of the $4^{\text {th }}$-order resonant inverter

These are defined with respect to the polarity of the input voltage, $V_{i n}$, and the state of the series resonant inductor current $i_{L S}$ and the parallel resonant capacitor voltage $V_{C p}$. However, for analysis purposes two dominant modes, $M 1$ and $M 2$ can be identified in each cycle with respect to the polarity of $V_{i n}$, as shown in Fig. 6. A state-variable description of the circuit behaviour can represented by the dynamics matrices $\mathbf{A}_{i}$ and the input excitation matrices, $\mathbf{B}_{i,}$ for each mode. In mode $M l, V_{\text {in }}>0$ and the piecewise linear state-equation is defined from the matrices:

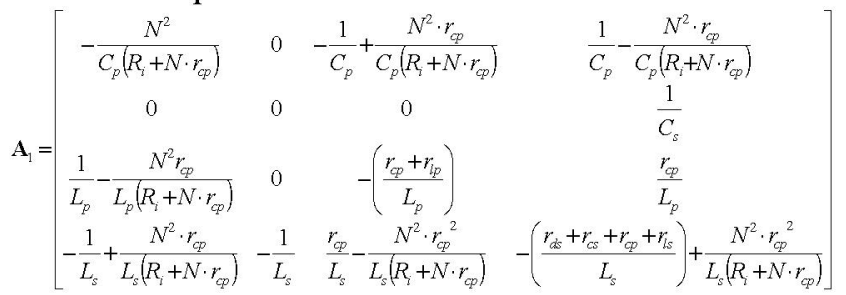

and, $\mathbf{B}_{1}=\left[\begin{array}{c}0 \\ 0 \\ 0 \\ V_{i n} \\ L_{s}\end{array}\right]$

Due to symmetry, the dynamics matrix $\mathbf{A}_{2}$ and the input matrix, $\mathbf{B}_{2}$, for inverter operation in mode $M 2\left(V_{i n}=0\right)$ is given by:

$$
\mathbf{A}_{2}=\mathbf{A}_{1} \text { and, } \mathbf{B}_{2}=\mathbf{0}_{4 \times 1}
$$

Since only two dominant modes are considered, depending on the polarity of the input voltage, symmetry dictates that the duty of each mode is $0.5 T_{s}$, where $T_{s}$ is the period for one cycle i.e. $d_{1}=0.5, d_{2}=0.5$. Substituting $(10,11)$ together with the duties, into $(8,9)$ therefore provides the initial operating condition of the circuit in steady state and an analytical solution for the circuit behaviour. An example phase portrait of the resonant circuit voltages and currents in steady state prior to ignition and during normal operation is shown in Fig. 7. It is notable that the computation time to obtain the analytical steady-state cyclic solution of the state vector is $\sim 1 / 10000 \times$ that required from Spice and other integration based simulation packages.

\section{CONCLUSIONS}

State-variable dynamic descriptions of a $4^{\text {th }}$-order resonant power inverter, used as fluorescent lamp ballast, have been presented.

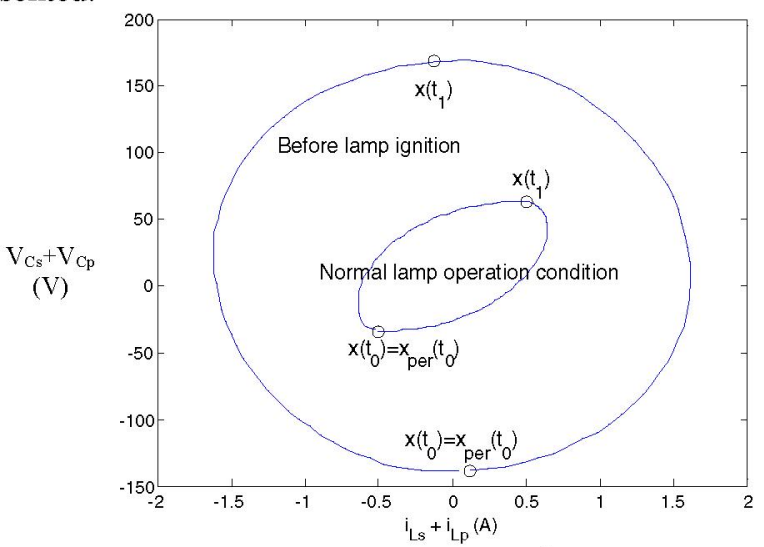

Figure 7 Steady state cyclic trajectory of the $4^{\text {th }}$-order resonant inverter during normal lamp operation, and prior to ignition of lamp

The model is subsequently used to obtain a steady-state cyclic description of the circuit, and the derivation of analytical formulae to calculate the electrical stresses on the resonant tank components. The accuracy of the proposed techniques has been shown by comparisons with both Spice simulations, and from measurements of an experimental $8 \mathrm{~W}$, capacitively coupled, fluorescent lamp, with good agreement being demonstrated. It is notable that, whilst the state-variable dynamic model requires a commensurate computational overhead to that of Spice simulations, the presented cyclic analysis method is typically $10^{4} \times$ faster.

\section{REFERENCES}

[1] D. O. Wharmby, 'Electrodeless lamps for lighting: a review, IEE Proceedings-A, vol. 140, pp. 465-473 Nov. 1993

[2] M. K. Kazimierczuk and W. Szaraniec, "Electronics Ballast for Fluorescent Lamps,' IEEE Trans. on Power Electronics, vol. 8, pp. 384395, Oct. 1993

[3] C. Chang, J. Chang, G. W. Bruning, 'Analysis of the SelfOscillating Series Resonant Inverters for Electronics Ballasts,' IEEE Trans. on Power Electronics, vol. 14, pp. 533-540, May 1999

[4] S. Y. R. Hui, L. M. Lee, H. Chung, Y. K. Ho, 'An Electronics ballast with Wide Dimming High PF, and Low EMI,' IEEE Trans. on Power Electronics, vol. 16, pp. 465-471, July 2001

[5] M. C. Cosby and R. M. Nelms, 'A Resonant Inverter for Electronics Ballast Applications,' IEEE Trans. on Industrial Electronics, vol. 41, pp. 418-425, Aug. 1994

[6] C. S. Moo, W. M. Chen, and H. K. Hsieh, 'Electronic Ballast with Piezoelectric Transformer for Cold Cathode Fluorescent Lamps,' IEE Procs. Electric Power Applications, vol. 150, pp. 278-282, March 2003

[7] L. R. Nerone, 'A Mathematical Model of the Class D Converter for Compact Fluorescent Ballast,' IEEE Trans. on Power Electronics, vol. 10, pp. 708-715, Nov. 1995

[8] S. Yaakov, M. Shvartsas, J. Lester, 'A Behavioural SPICE Compatible Model of an Electrodeless Fluorescent Lamp,' APEC, 2002, pp. 948-954

[9] J. Alonso, C. Blanco, E. Lopez, A. J. Calleja, and M. Rico, 'Analysis, Design, and Optimization of the LCC Resonant Inverter as a High-Intensity Discharge lamp Ballast,' IEEE Trans. on Power Electronics, vol. 13, pp. 573-585, May 1998

[10] M. K. Kazimierczuk, Resonant Power Converters. New York : John Wiley and Son, 1995 
[11] H. R. Visser and P. P. J. Borch, 'Modelling of Periodically Switching Networks,' PESC 91 Records $22^{\text {nd }}$ IEEE Power Electronics Specialists Conference, 1991, pp. 67-73

[12] Y. A. Ang, D. A. Stone, C. M. Bingham and M. P. Foster, 'Analysis and Design of High-frequency LCLC Converters for Electrodeless Fluorescent Lamp Ballast,' in press for PEMD 2004

[13] M. P. Foster, H. I. Sewell, C. M. Bingham, D. A. Stone, D. Hente \& D. Howe: 'Cyclic-averaging for high-speed analysis of resonant converters.' IEEE Transactions on Power Electronics, vol. 18, pp. 985993, July 2003.

[14] Y. A. Ang, M. P. Foster, C. M. Bingham, D. A. Stone, H. I. Sewell, D. Howe: 'Analysis of $4^{\text {th }}$-order LCLC Resonant Power Converters.' IEE Electric Power Applications, vol. 151, pp. 169-181, March 2004 